\title{
Glucagon-like peptide-1 (GLP-1) stimulates insulin secretion and then somatostatin secretion in rat islets.
}

\section{Seungjoon Oh ${ }^{1,2}$, So Young Park ${ }^{2}$, Kwang Sik Suh², Sung-Woon Kim ${ }^{1.2}$}

'Department of Endocrinology and Metabolism, Kyung Hee University School of Medicine; ${ }^{2}$ Research Institute of Endocrinology, Kyung Hee University Hospital, Seoul, Korea

\section{Abstract}

- Objective : GLP-1 is known to stimulate insulin and somatostatin secretion in pancreatic islet cells. The exogenous somatostatin inhibits insulin secretion, but GLP-1 increases insulin secretion in spite of stimulating somatostatin secretion from d-cell. So, we investigated whether there exists a time difference of insulin and somatostatin secretion after GLP-1 stimulation or insulin secretion depends on secreted GLP-1 or somatostatin concentration inside the islets.

- Methods: We isolated pancreatic islets from five 8-week-old Sprague Dawley rats by collagenase digestion. The islets were incubated in RPMI1640 medium before experiments. In vitro, insulin and somatostatin measured at $5,10,30$ minutes depending on glucose $(2.7,5.5$ and $16.7 \mathrm{mM}$ as hyponormo-, and hyperglycemic condition respectively) and GLP-1 concentrations $(0,0.1$, and $10 \mathrm{ng} / \mathrm{mL}$ ) from culture media using ELISA kit. Each well contained 30 islets per well.

- Results: Insulin secretion showed continuously increased by time and GLP-1 concentration at any glucose concentration. Somatostatin secretion was increased significantly 10 minutes later after GLP-1 administration at hypo- and normoglycemia. However, in hyperglycemia, insulin secretion showed the same pattern compared with the other glycemic conditions, but somatostatin was maximally stimulated until 10 minutes and decreased without GLP-1 administration. Adding the GLP-1, somatostatin was increased continuously until 30 minutes. This suggested hyperglycemic condition itself might need more insulin secretion in the $B$-cell than somatostatin secretion in d-cell. Therefore, we observed time lag of intraislet somatostatin secretion after GLP-1 stimulation.

- Conclusion: We observed earlier GLP-1 induced insulin secretion in B-cell than somatostatin secretion in $\mathrm{d}$-cell of rat islets. Hyperglycemic condition might primarily secrete insulin and inhibit somatostatin secretion inside the islets.

\section{Background}

E GLP-1 is known to stimulate insulin and somatostatin secretion in pancreatic islet cells.

E The exogenous somatostatin inhibits insulin secretion. But GLP-1 increases insulin secretion in spite of stimulating somatostatin secretion from beta-cell.

E Purpose

existence of a time difference of insulin and somatostatin secretion inside the islets after GLP-1 stimulation

insulin secretion rate in accordance with GLP-1 or somatostatin concentration inside the pancreatic islet cells

\section{Materials and Methods}

E The pancreatic islets were isolated form 8-week-old Sprague Dawley rats by collagenase digestion and incubated in RPMI 1640 medium.

E In vitro, insulin and somatostatin measured at 5, 10,30 minutes depending on

* glucose concentration

- Hypoglycemia $(2.7 \mathrm{mM})$

Normoglycemia $(5.5 \mathrm{mM})$

- Hyperglycemia (16.7 mM)

GLP-1 concentration

$.0 \mathrm{ng} / \mathrm{mL}$

- $0.01 \mathrm{ng} / \mathrm{mL}$

- $10 \mathrm{ng} / \mathrm{mL}$

- 30 islets per well

E Analysis with ELISA kit

\section{Results}

- Hypoglycemia (2.7 mM) (Table 1, Figure 1A)

* Insulin secretion

: continuously increased by time and GLP-1 concentration

* Somatostatin secretion

: increased significantly 10 minutes later after GLP-1 administration.

Normoglycemia $(5.5 \mathrm{mM})$ (Table 2, Figure 1B)

* Insulin secretion

continuously increased by time and GLP-1 concentration

* Somatostatin secretion

increased significantly 10 minutes later after GLP-1 administration.

Hyperglycemia (16.7 mM) (Table 3, Figure 1C)

* Insulin secretion

: continuously increased by time and GLP-1 concentration.

* Somatostatin secretion

: maximally stimulated until 10 minutes and decreased without GLP-1 administration. : increased continuously until 30 minutes after GLP-1 administration.

Table 1. In hypoglycemia, insulin and somatostatin concentration depending on GLP-1.

\begin{tabular}{|c|c|c|c|c|}
\hline \multicolumn{5}{|c|}{ Hypoclycemia ( glucose $2.7 \mathrm{mM}$ ) } \\
\hline & & $5 \mathrm{~min}$ & $10 \mathrm{~min}$ & $30 \mathrm{~min}$ \\
\hline \multirow{2}{*}{ GLP-1 0 ng/mL } & Insulin(mU/mL) & 78.4 & 99.5 & 126.7 \\
\hline & Somatostatin $(\mathrm{pg} / \mathrm{mL})$ & 0.28 & 0.67 & 2.02 \\
\hline \multirow{2}{*}{ GLP-1 $0.1 \mathrm{ng} / \mathrm{mL}$} & Insulin(mU/mL) & 86.7 & 100.3 & 124.4 \\
\hline & Somatostatin $(\mathrm{pg} / \mathrm{mL})$ & 0.99 & 0.42 & 2.07 \\
\hline \multirow{2}{*}{ GLP-1 $10 \mathrm{ng} / \mathrm{mL}$} & Insulin(mU/mL) & 89.1 & 112.3 & 130.5 \\
\hline & Somatostatin $(\mathrm{pg} / \mathrm{mL})$ & 1.26 & 0.84 & 3.06 \\
\hline
\end{tabular}

Table 2. In normoglycemia, insulin and somatostatin concentration depending on GLP-1.

\begin{tabular}{|c|c|c|c|c|}
\hline \multicolumn{5}{|c|}{ Normoglycemia ( glucose $5.5 \mathrm{mM}$ ) } \\
\hline & & $5 \mathrm{~min}$ & $10 \mathrm{~min}$ & $30 \mathrm{~min}$ \\
\hline \multirow{2}{*}{ GLP-1 0 ng/mL } & Insulin $(\mathrm{mU} / \mathrm{mL})$ & 94.2 & 116.4 & 145.8 \\
\hline & Somatostatin(pg/mL) & 0.4 & 0.71 & 2.67 \\
\hline \multirow{2}{*}{ GLP-1 $0.1 \mathrm{ng} / \mathrm{mL}$} & Insulin(mU/mL) & 100.3 & 121.8 & 149.3 \\
\hline & Somatostatin(pg/mL) & 0.42 & 0.81 & 3.74 \\
\hline \multirow{2}{*}{ GLP-1 $10 \mathrm{ng} / \mathrm{mL}$} & Insulin(mU/mL) & 120.3 & 135.2 & 174.7 \\
\hline & Somatostatin(pg/mL) & 0.84 & 0.86 & 5.31 \\
\hline
\end{tabular}

Table 3. In hyperglycemia, insulin and somatostatin concentration depending on GLP-1.

\begin{tabular}{|c|c|c|c|c|}
\hline \multicolumn{5}{|c|}{ Hyperglycemia ( glucose $16.7 \mathrm{mM}$ ) } \\
\hline & & $5 \min$ & $10 \mathrm{~min}$ & $30 \mathrm{~min}$ \\
\hline \multirow{2}{*}{ GLP-1 $0 \mathrm{ng} / \mathrm{mL}$} & Insulin(mU/mL) & 120.3 & 136.7 & 185.7 \\
\hline & Somatostatin $(\mathrm{pg} / \mathrm{mL})$ & 1.25 & 4.47 & 3.17 \\
\hline \multirow{2}{*}{ GLP-1 $0.1 \mathrm{ng} / \mathrm{mL}$} & Insulin(mU/mL) & 124.4 & 144.2 & 195.3 \\
\hline & Somatostatin $(\mathrm{pg} / \mathrm{mL})$ & 2.07 & 3.4 & 4.54 \\
\hline \multirow{2}{*}{ GLP-1 $10 \mathrm{ng} / \mathrm{mL}$} & Insulin(mU/mL) & 130.5 & 156.5 & 221.4 \\
\hline & Somatostatin $(\mathrm{pg} / \mathrm{mL})$ & 3.06 & 4.88 & 7.09 \\
\hline
\end{tabular}

Hypoglycemia Glucose, $2.7 \mathrm{mM}$

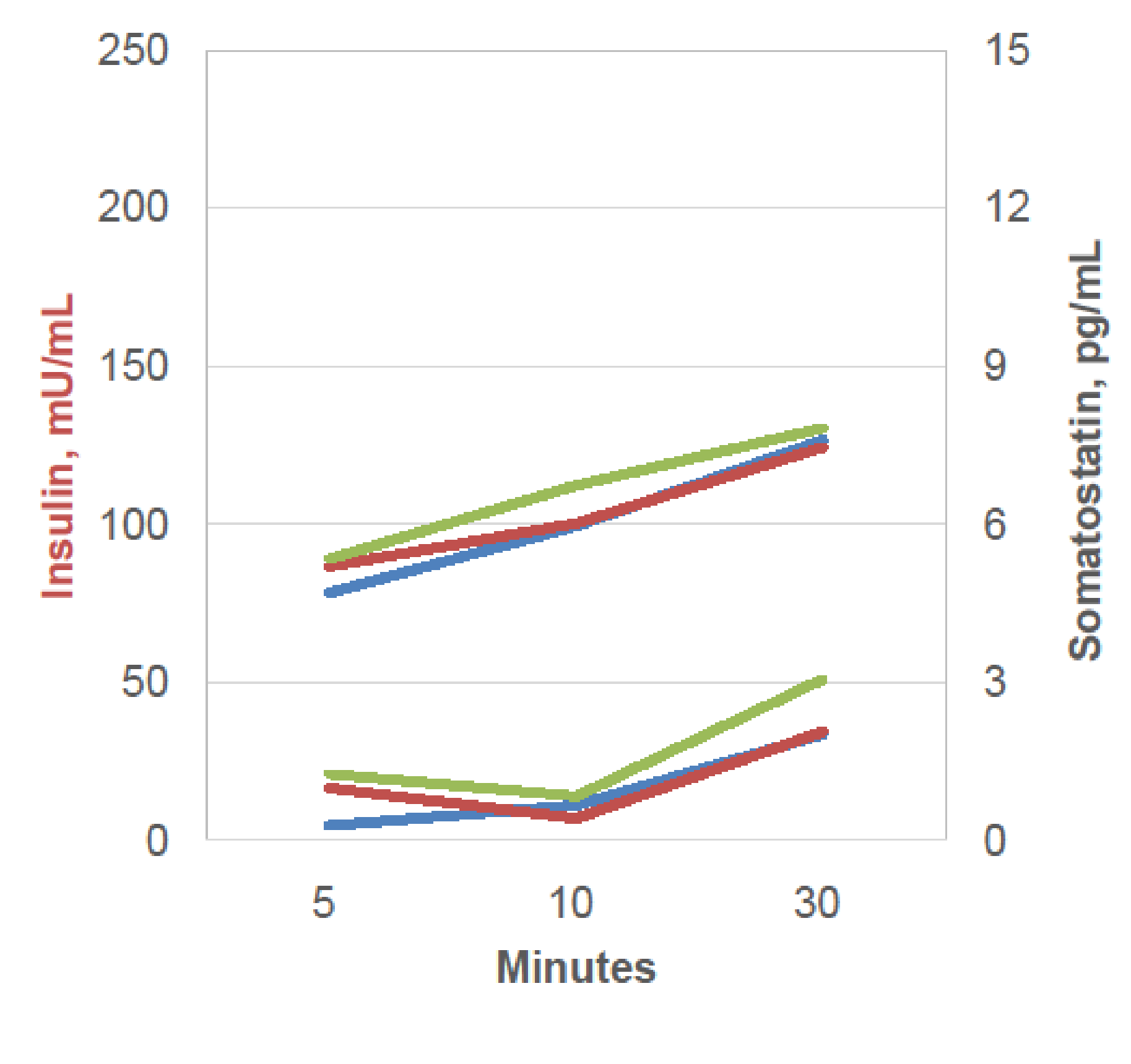

Hyperglycemia Glucose, $16.7 \mathrm{mM}$

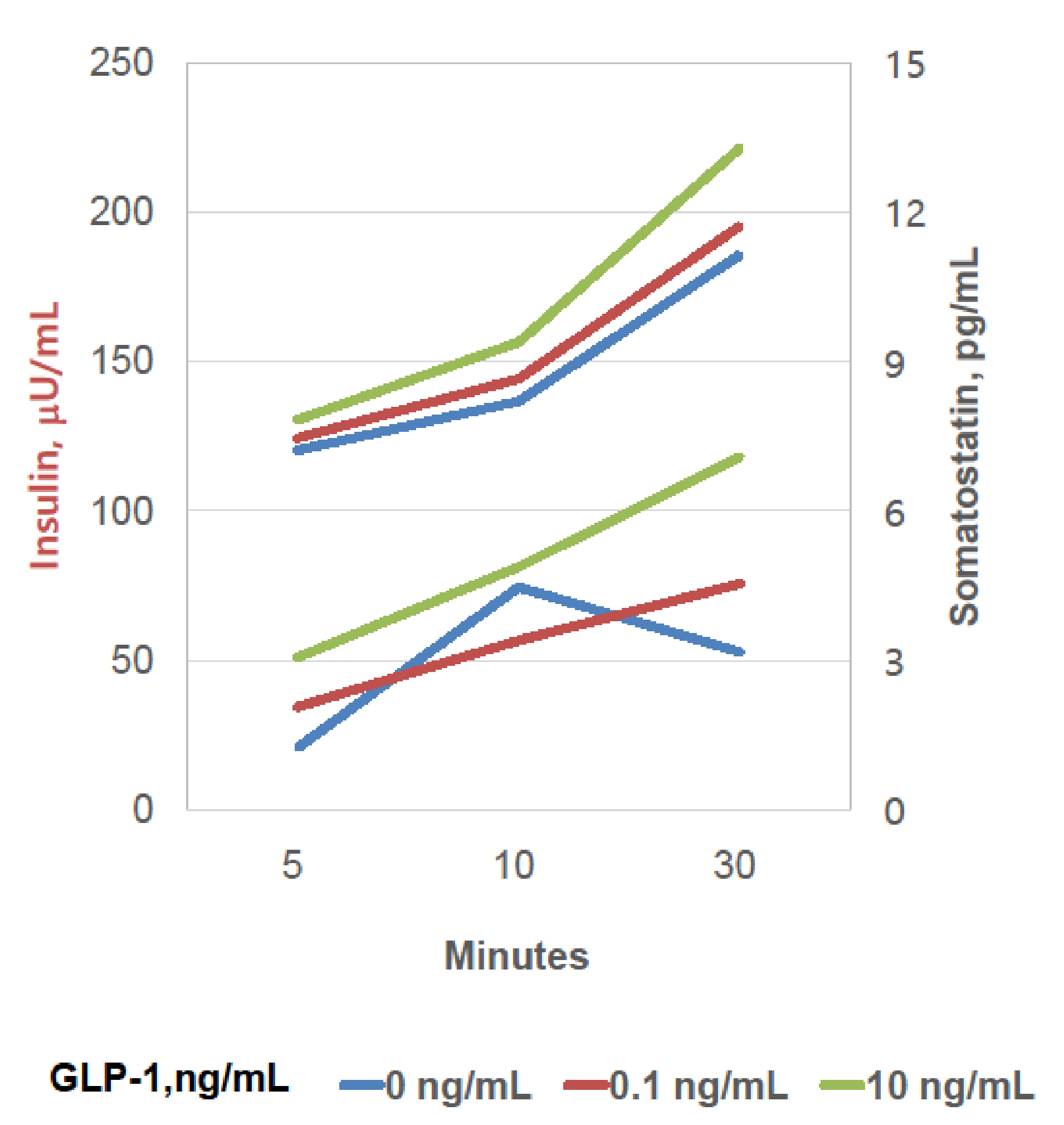

C

Figure 1. Insulin and Somatostatin concentration depending on GLP-1. hypoglycemia (A) normoglycemia(B), hyperglycemia(C), somatostatin secretion rate depending on GLP-1 and glucose concentraion.

\section{Conclusion}

E Hyperglycemic condition itself might need more insulin secretion in the $ß$-cell than somatostatin secretion in $\alpha$-cell.

E GLP-1 induced insulin secretion in $\beta$ cell is earlier than somatostatin secretion in a-cell of rat islets.

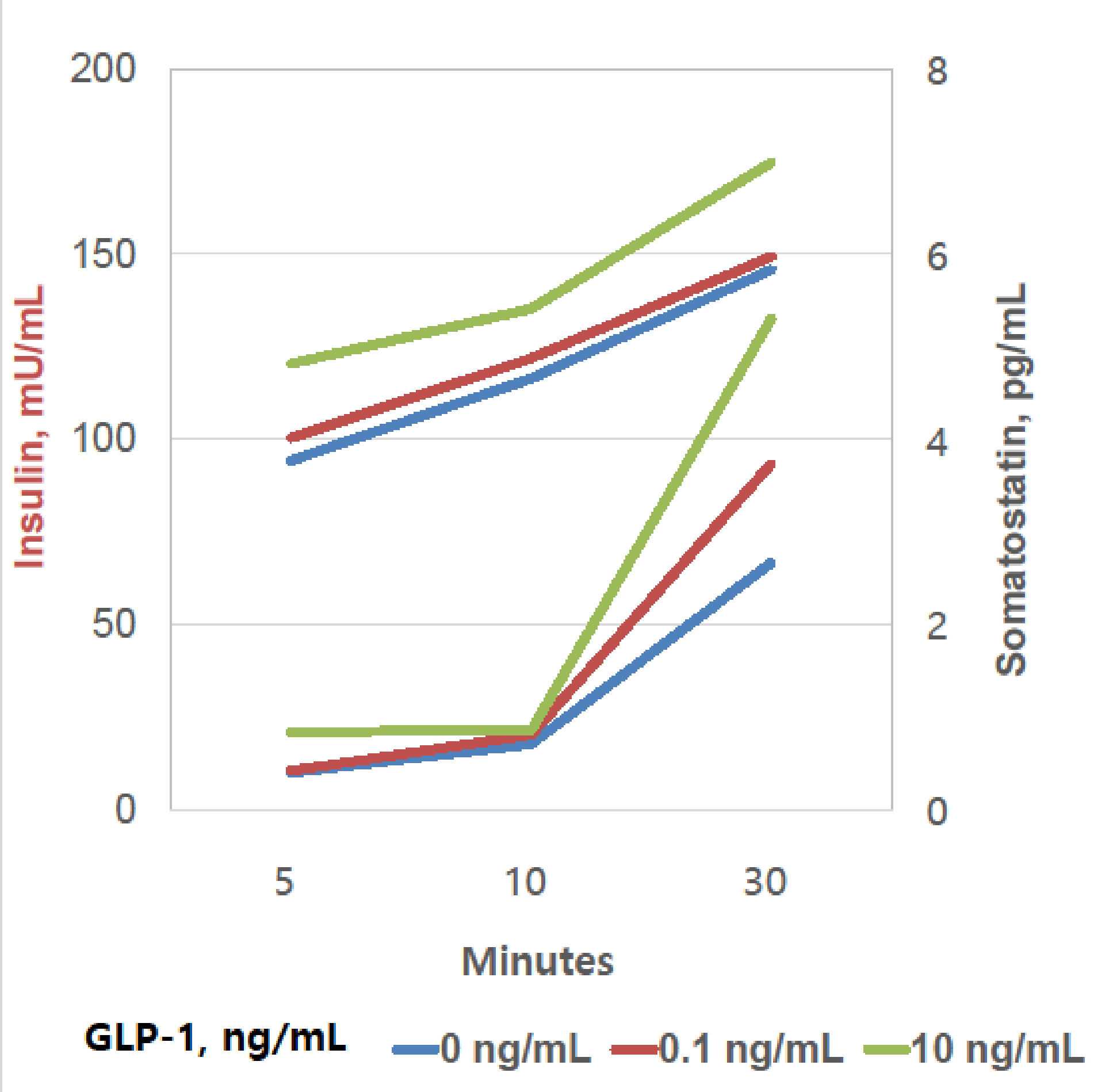

B

Somatostatin, $\mathrm{pg} / \mathrm{mL}$

GLP-1,ng/mL Normoglycemia, $5.5 \mathrm{mM}-\mathrm{N}-\mathrm{N}-\mathrm{N}$

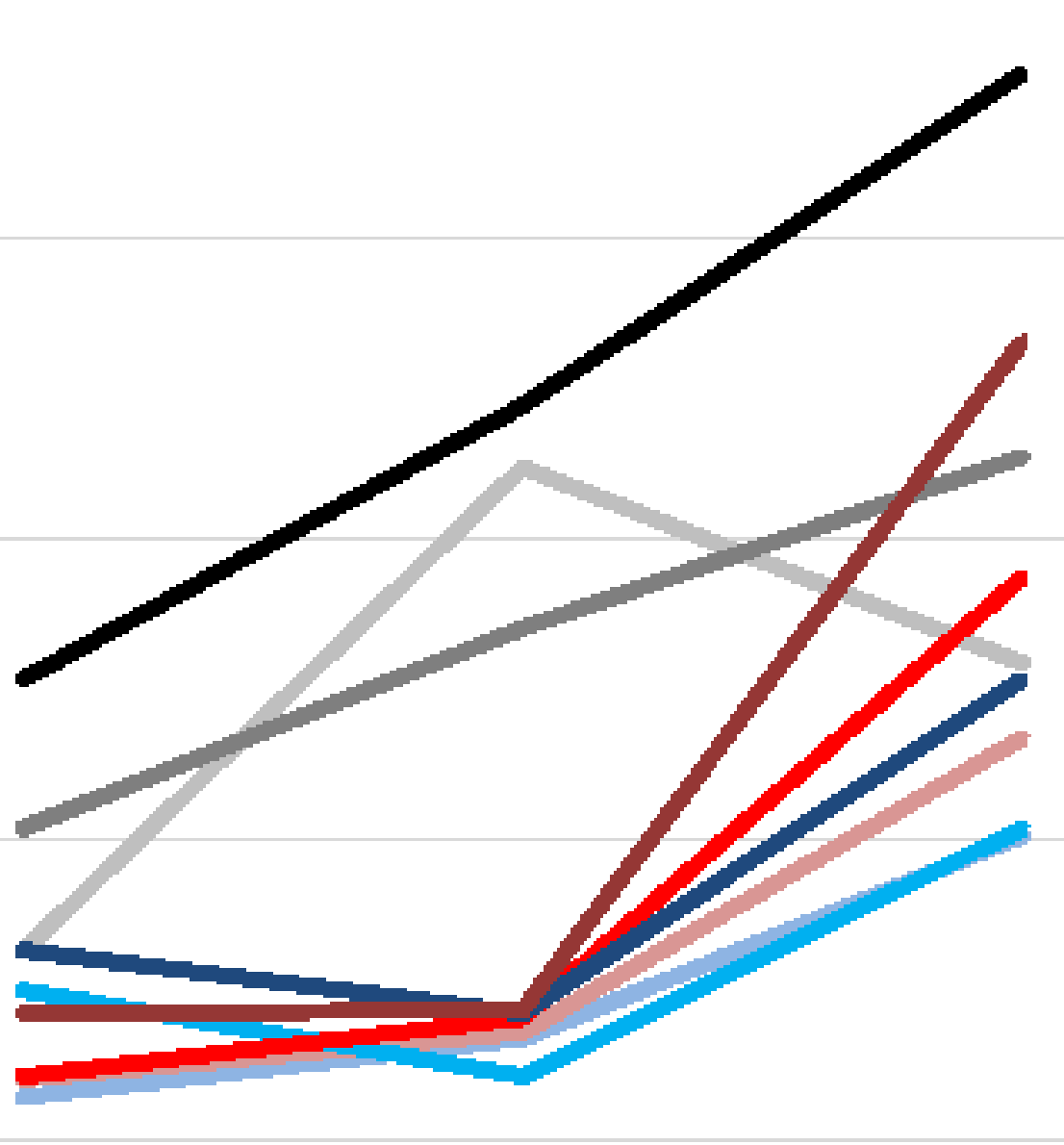
d 